\title{
ATLAS Magnet Common Cryogenic, Vacuum, Electrical and Control Systems
}

\author{
P. Miele, F. Cataneo, N. Delruelle, C. Geich-Gimbel, F. Haug, G. Olesen, R. Pengo, E. Sbrissa, H. Tyrvainen, and \\ H. H. J. ten Kate
}

\begin{abstract}
The superconducting Magnet System for the ATLAS detector at the LHC at CERN comprises a Barrel Toroid, two End Cap Toroids and a Central Solenoid with overall dimensions of $20 \mathrm{~m}$ diameter by $26 \mathrm{~m}$ length and a stored energy of $1.6 \mathrm{GJ}$. Common proximity cryogenic and electrical systems for the toroids are implemented. The Cryogenic System provides the cooling power for the 3 toroid magnets considered as a single cold mass (600 tons) and for the CS. The $21 \mathrm{kA}$ toroid and the 8 kA solenoid electrical circuits comprise both a switch-mode power supply, two circuit breakers, water cooled bus bars, He cooled current leads and the diode-resistor ramp-down unit. The Vacuum System consists of a group of primary rotary pumps and sets of high vacuum diffusion pumps connected to each individual cryostat. The Magnet Safety System guarantees the magnet protection and human safety through slow and fast dump treatment. The Magnet Control System ensures control, regulation and monitoring of the operation of the magnets. The updated design, layout, development and construction of the systems, as well as the first results of prototyping and commissioning are presented.
\end{abstract}

Index Terms-Cryogenic, magnet, superconductor, toroid.

\section{INTRODUCTION}

$\mathbf{T}$ HE ATLAS Magnet System [1]-[3] consists of the superconducting magnets and the related services, such as power supply, cryogenic, vacuum, control and safety systems. The magnet system has overall dimensions of 26 meters in length and 20 meters in diameter, Fig. 1. The superconducting magnets comprise a Barrel Toroid (BT), two End-Cap Toroids (ECT) and the Central Solenoid (CS). Each of the 3 toroids consists of 8 coils, with two double pancake windings embedded in an aluminum alloy casing, assembled around the beam axis with an offset angle of $22.5^{\circ}$ between the BT and ECT systems, in order to optimize the particle bending power in the available space. The CS is a single layer coil wound internally in a supporting cylinder and enclosed by the common cryostat of the Liquid Argon Calorimeter. The coils are built with Aluminum stabilized $\mathrm{NbTi} / \mathrm{Cu}$ superconductor, insulated with glass tape and fully impregnated with epoxy resin. An Al thermal shield and the superinsulation layers surround the cold mass. A complete cryogenic system supplies liquid and gaseous Helium at $4.5 \mathrm{~K}$ and $60 \mathrm{~K}$ for cooling of the cold mass and thermal shield respectively. The electrical system consists of a $21 \mathrm{kA}$ common toroid power system and an $8 \mathrm{kA}$ solenoid power system and related quench protection systems.

Manuscript received October 20, 2003.

The authors are with CERN, 1211 Geneve 23, Switzerland (e-mail: paola.miele@cern.ch).

Digital Object Identifier 10.1109/TASC.2004.829704

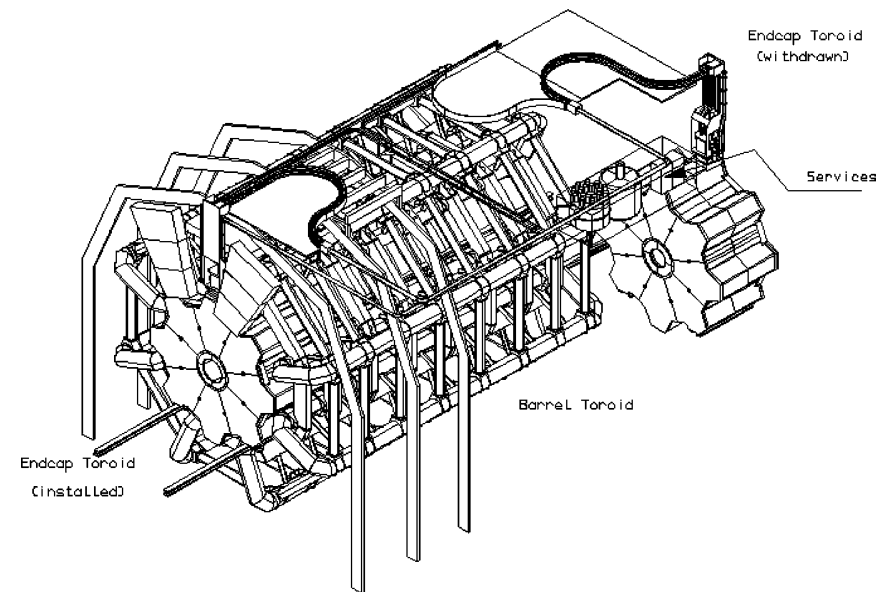

Fig. 1. The ATLAS Magnet System showing the three toroids.

The BT [4] constitutes the main structure of the ATLAS Magnet System. Each coil has its own vacuum vessel, which is connected one to neighboring coils through the cryogenic ring enclosing the electrical and cryogenic interconnections. The warm structure with its supporting rings constitutes the mechanical support structure of the system. The whole assembly is supported on the feet. The two ECT [5], Fig. 1, are inserted in the BT at each end of the CS. Each ECT contains 8 racetrack coils mounted as a single cold mass in a common vacuum vessel. All services are connected through a single service turret mounted on top of the vacuum vessel. Each ECT is mounted on the main rail structure to allow movement for access to the inner part of the detector. The CS [6] with a length of $5.3 \mathrm{~m}$ and a bore of $2.5 \mathrm{~m}$, provides $2 \mathrm{~T}$ axial magnetic field in the central tracking volume of the ATLAS detector. The superconducting bus bars and cryo-lines connect the CS coil through a $12 \mathrm{~m}$ long chimney to the proximity cryogenics positioned on top of the detector.

\section{THE SERVICES}

The magnets require for their functioning a number of services providing vacuum, cryogenic cooling, electrical power, control and safety, which are organized at CERN through contracts with specialized companies. Standard services, such as air and water, ethernet/field bus, mains and UPS, are mainly provided by CERN support groups. The cost of the service systems is minimized, while maintaining high technical performance and reliability. To decrease the complexity of the distribution 


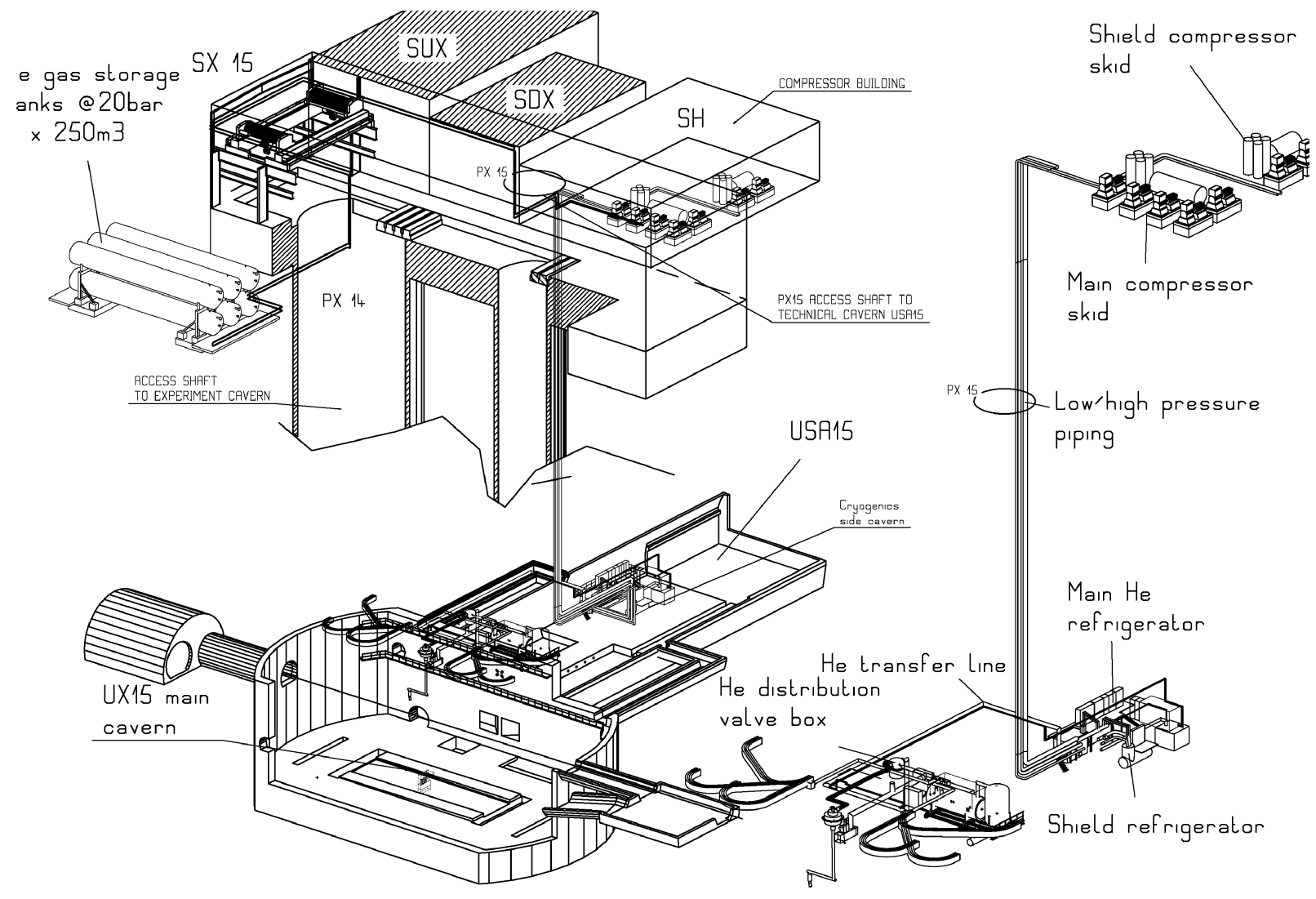

Fig. 2. The cryogenic system: the external and proximity cryogenics.

lines and therefore the cost and to increase the overall reliability of the system a common proximity cryogenic and electrical system for the toroids is implemented.

\section{A. Cryogenic System}

The cryogenic system consists of:

1) Internal Cryogenics: the cooling circuit of the cold mass and thermal shield, with full redundancy;

2) Proximity Cryogenics: the auxiliary cryogenic equipment necessary for the operation of each magnet, i.e. the toroids, which are cooled as a single cold mass (600 tons) and the solenoid (6 tons). The toroid system requires $1200 \mathrm{~g} / \mathrm{s}$ of two-phase $\mathrm{He}$ at $4.5 \mathrm{~K}$, which is circulated by means of 2 centrifugal pumps and related distribution unit, while the solenoid requires only $7 \mathrm{~g} / \mathrm{s}$ of $4.5 \mathrm{~K} \mathrm{He}$ taken directly from the refrigerator.

3) External Cryogenics: the He refrigerator plants and related distribution system, common to all magnets.

The refrigeration system [7], Fig. 2, comprises two cryoplants: the Shield Refrigerator and the Main Refrigerator. The shield refrigerator is used for the cool-down phase between $300 \mathrm{~K}$ and $100 \mathrm{~K}$ with a cooling capacity of $60 \mathrm{~kW}$ provided by a liquid nitrogen/helium heat exchanger, and to provide in steady-state shield cooling, which requires a nonisothermal capacity of $20 \mathrm{~kW}$ between $40 \mathrm{~K}$ and $80 \mathrm{~K}$. The single stage compressors have been installed, commissioned and accepted. The main refrigerator is used for the final cool-down phase from $100 \mathrm{~K}$ to $4.5 \mathrm{~K}$ and the steady-state operation of the magnet cold masses at $4.5 \mathrm{~K}$. This refrigerator has an equivalent cooling power of $6 \mathrm{~kW}$ at $4.5 \mathrm{~K}$. Parallel cool down with a master/slave modality is chosen to control the system. Three Helium transfer lines, the He distribution valve box providing LHe to both toroid and solenoid proximity cryogenics, the $250 \mathrm{~m}^{3}$ GHe buffer tanks (6) and the $\mathrm{LN}_{2}$ dewars (2) with related transfer lines complete the external cryogenics. The external cryogenics is now being installed and commissioning will be completed by April 2004.

The solenoid proximity cryogenics [8] consists of the control dewar and valve unit at the top of the detector connected to the coil by a $12 \mathrm{~m}$ long chimney. It has been successfully tested at Toshiba and CERN. A current of $9 \mathrm{kA}$ was applied, which corresponds to $20 \%$ margin on the nominal value of $7.4 \mathrm{kA}$.

The toroid Proximity Cryogenic System [9] comprises a valve and phase separator main box containing the majority of the control elements (valves, flow meters, pressure and temperature sensors) and the heat exchanger to remove extra power to the fluid exiting the pumps, the He pump cryostat with two $1200 \mathrm{~g} / \mathrm{s}$ centrifugal pumps for redundancy. The buffer/storage dewar with a capacity of 110001 allows the slow discharge during 2 hours in case of failure of the external cryogenics. In addition it includes the BT current lead cryostat, the fixed and flexible cryogenic transfer lines and the gas control panel. Test of the $1200 \mathrm{~g} / \mathrm{s}$ (about $10 \mathrm{l} / \mathrm{s}$ ) pump was successfully performed at CERN: Fig. 3 shows the characteristic curve and the pump efficiency, both complying with the requirements ( $400 \mathrm{mbar}$ at $1.2 \mathrm{~kg} / \mathrm{s}$, total efficiency $\sim 60 \%$ ) [10]. This is the largest LHe pump ever built. 


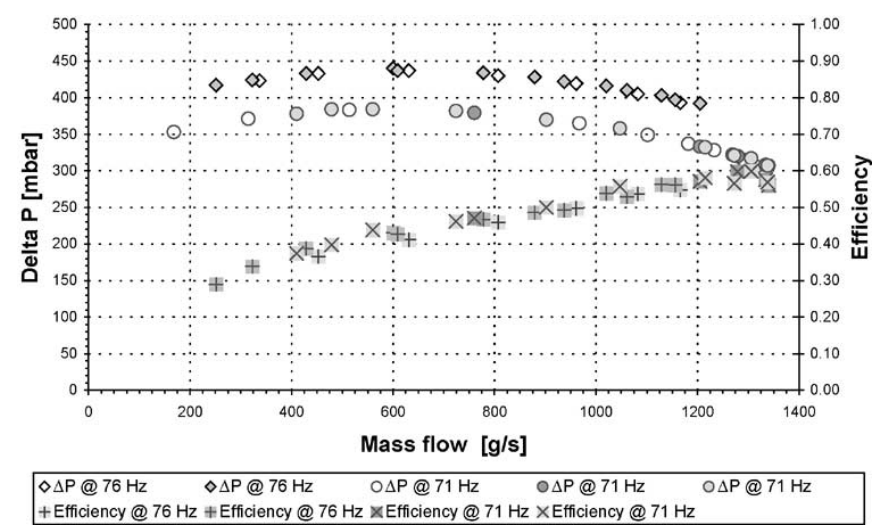

Fig. 3. Characteristic curves and efficiencies of the pump.

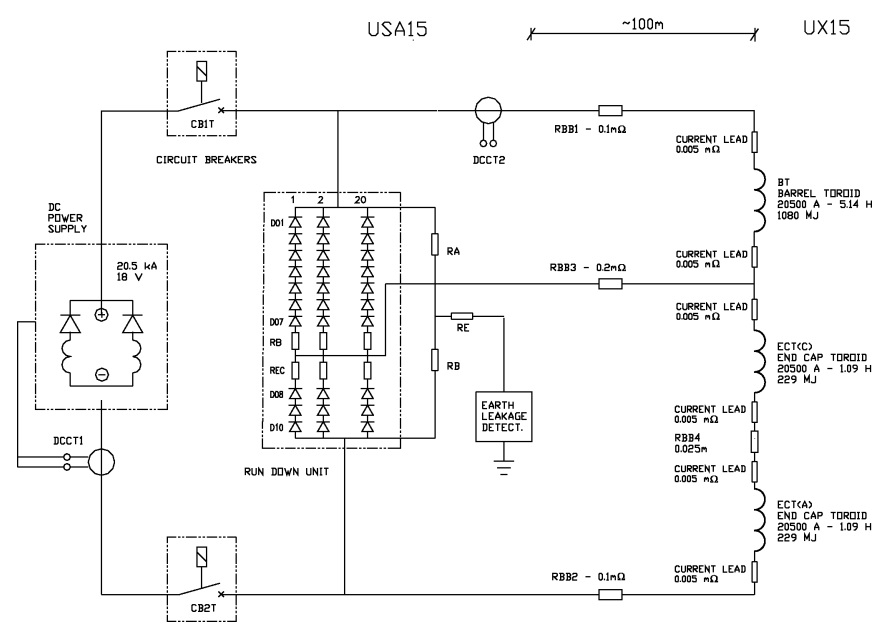

Fig. 4. The toroid electrical system.

\section{B. Electrical System}

The toroid electrical circuit, Fig. 4, comprises the $21 \mathrm{kA}$, $20 \mathrm{~V}$ switched-mode power supply, two DCCT's, two circuit breakers, $\sim 300 \mathrm{~m}$ bus bars, He cooled current leads, earth leakage detection system and the diode/resistor ramp-down unit connected across the coils. The ramp-up time of the toroids is 2-3 hours, with an initial ramp rate of $4 \mathrm{~A} / \mathrm{s}$. The solenoid has a separate $8 \mathrm{kA}$ power supply due to its different current rating. High frequency $(20-50 \mathrm{kHz})$ switching techniques are used to avoid the weight and size of conventional thyristor supplies. DCCT1 is used for the regulation of the power supply and DCCT2 for monitoring the magnet current. Reliability of the ramp-down unit is based on a large number of diode/resistor circuits connected in parallel ( 20 for the toroids and 10 for the CS). The bus bars operate safely without cooling, however cooling by demineralized water is needed to avoid heat dissipation in the cavern atmosphere. Two circuit breakers, one for each polarity, are used to increase reliability and safety of slow dump and possibly isolate the power supply for test and maintenance purpose. Production of the components is ongoing and installation starts in October 2003 with the bus bars in the service cavern.

\section{Magnet Safety System}

The Magnet Safety System insures the magnet protection in stand alone mode with fast and slow discharge request. The coils are de-energized through a diode/resistor ramp-down unit. In the case of a quench the stored energy is dumped in the magnet cold mass by means of quench heaters embedded in all coils. A low external dump resistor $<2 \mathrm{~m} \Omega$ reduces current and force imbalance in the unlikely case of short circuits in the coils. A new Superconductor Quench Detector (SQD) is developed to detect a quench in the bus bar with high resolution (one SQD at each bus bar section between double pancakes and at the current lead feet). In addition the protection of the magnet relies on 3 bridge detection systems based on unbalanced voltage readings through the magnet, and differential quench detection based on differential voltage between double pancakes and compensation coil. For safety reasons 48 VDC Uninterrupted Power Supply (UPS) is used to guarantee the functionality of the system in any fault condition. Redundancy is foreseen for all safety sensors, detection channels and quench heaters.

\section{Magnet Control System}

The Magnet Control System provides the process control to ensure in full automatic mode the operating sequences of the magnets and the control, regulation and monitoring of all the functional parameters related to the magnets and to the cryogenic, electrical and vacuum systems. Harmonization and standardization requirements for all the LHC experiments are followed for operational and maintenance easiness. It consists of sensors and actuators, Programmable Logic Controllers (PLC), the magnet supervisor and fast and slow Data Acquisition Systems. High level communication is through a standard industrial field-bus, while all sensor and actuator connections and safety system are hard-wired. A functional description of the modes of operation and related processes, including fault scenario, is the basis for the control development, to be translated in UNICOS (Unified Industrial Control System) standard. Schneider PLC's and PVSS (Process Visualization and Supervision System) are the standard tools for the whole control system. Fig. 5 shows the control architecture with related Data Exchange Network enabling communication between the systems and the control rooms for commissioning and operation.

\section{E. Vacuum System}

The 8 separate cryostats of the BT are connected through the common cryoring to make one vacuum, while two separate cryostats contain the 8 coils of the ECT's. A common toroid primary system with single and two stage rotary pumps is located on the floor of the cavern and the high vacuum system comprises 21 diffusion pumps, 2 at each BT coil (16 total), 2 at each ECT ( 4 total) and 1 at the proximity cryogenics. The design guarantees redundancy and when fully operational satisfies the vacuum level even in the case of small leaks. No turbomolecular pumps could be used because they are not compatible with radiation and magnetic field. Restrictors are used to reduce the pump inlet pressure with respect to the vessel pressure and fill in the gap between $10^{-1}$ and $10^{-2}$ mbar required to make the diffusion pumps work. The test setup, Fig. 6 , allows to verify 


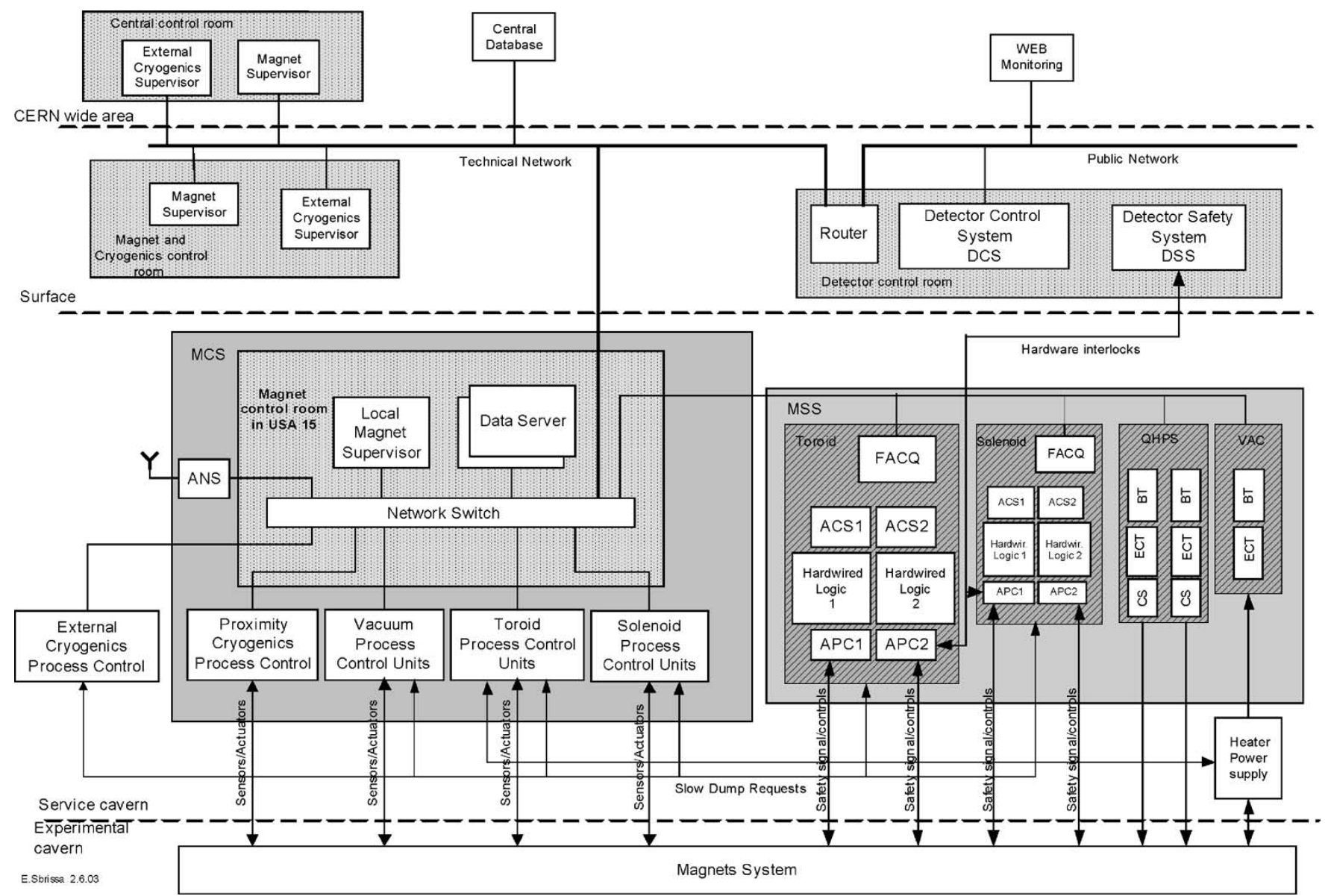

Fig. 5. Control architecture.

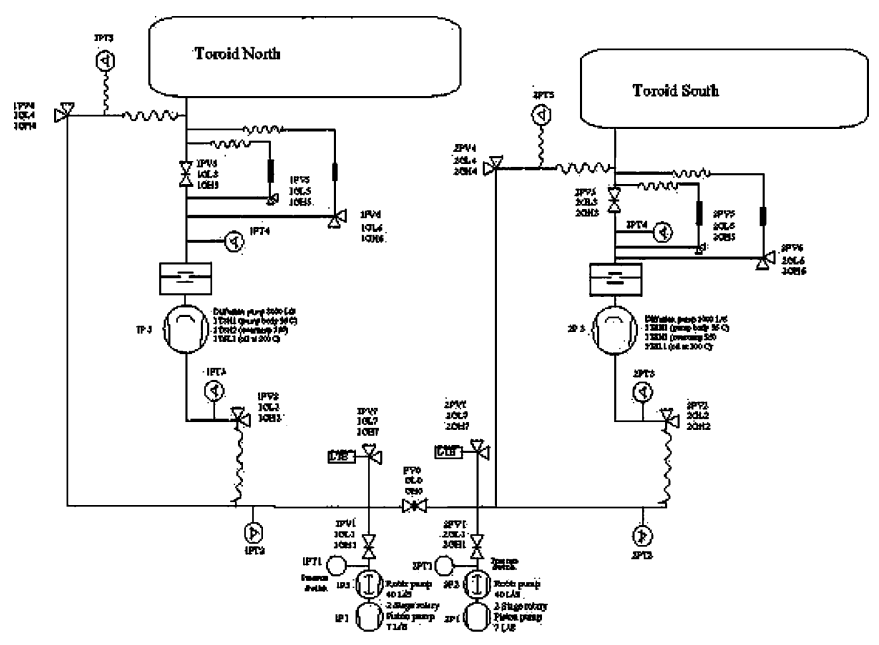

Fig. 6. Vacuum test setup with the bypass scheme.

the performance of the bypass scheme, simulating the coil outgassing with a dose valve. Fig. 7 shows the pressure ranges when pumping through the restrictors compared with the full cross section.

\section{FAULT SCENARIOS}

An analysis of the fault scenario for the magnets and the common systems is at the basis of the design. Electrical faults comprise short and open circuit. External short circuit faults

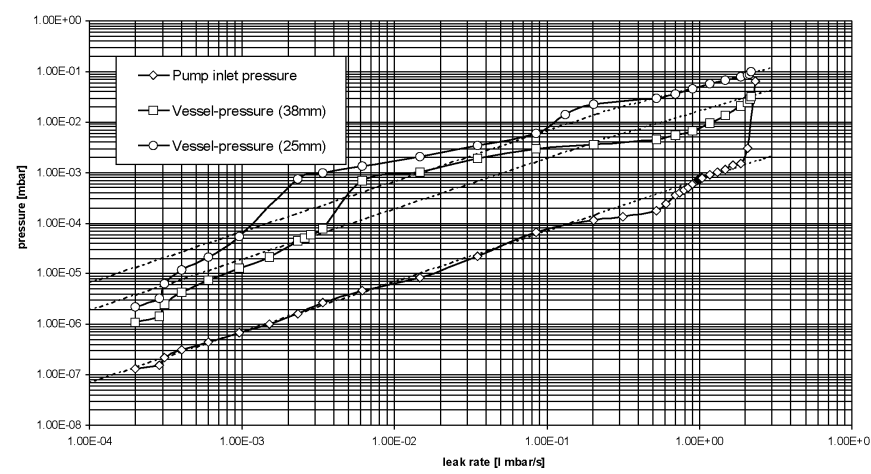

Fig. 7. Pressure ranges with restrictors of 25 and $38 \mathrm{~mm}$ diameter.

may arise in the busbar system. Depending on the position of the short circuit it may affect the possibility to discharge the magnets safely. Open circuit faults can arise due to the conductor burnout, current lead burnout or mechanical interruption of the external busbar system. Good insulation and protection across the whole line are executed in particular at the joint area, i.e. at the cryoring bus, the current lead joints and the conductor joints. A weak point is the connection between the bus bars and the current leads, where the contrasting request of flexibility for thermal shrinkage and rigid support must be guaranteed. Voltage limiters for $2 \mathrm{kV}$ (spark gap or current surge suppressor) at the bus bars are implemented to avoid coil burnout in the case of open circuit. The main impact of short circuit is expected during 
magnet quench or fast dump actions, resulting in unbalanced currents and forces internally and between toroids.

Cryogenic/vacuum faults derive from a serious leak in the internal cryogenic circuit releasing $\mathrm{He}$ cryogen or a major vacuum leak allowing rapid ingress of air. The effect is a rapid failure of the cryostat vacuum with high heat leak between the magnet cold mass and vessel. Cooling of the vessel wall leads to increased mechanical stresses and water condensation on the vessel outer surface, which has serious consequences for the operation of the detector. The solution adopted consists in industrial heaters on the vessel surface. Calculations and tests were done to optimize this solution. Redundancy of the heater circuits and of the power sources guarantees the correct functioning of this solution. A leak in the cryogenic circuitry is one of the events to be strictly avoided: redundant cooling systems on the cold mass and thermal shield and very high quality requirements on the welds of the lines minimize the risk. In the case of electrical/ compressed air failure the Fail Safe Position applies to all valves. Safety relief valves guarantee against overpressure in the cryogenic lines and in the vacuum vessel. At the Buffer Dewar and Phase Separator the safety relief valves are fully redundant and acting on different principle (mechanical and magnetic). Control and safety system faults may have serious knock-on effects e.g. failure of the quench detector system or quench heater system. The MSS is extremely robust in terms of redundancy and safety factors; in addition two quench detection systems based on different principle of detection are implemented to increase the intrinsic safety. Moreover all cables are hard wired and redundant channels are separated.

\section{CONCLUSION}

Proper operation of the common service systems has to guarantee the correct operation of the whole ATLAS Magnet
System. The design of the common systems is complete and construction is well advanced. The design follows standardization and harmonization requirements with the LHC machine. Installation and commissioning of all the common systems must be completed by March 2005, to allow for testing of the BT magnet. The cost is minimized while maintaining optimized technical performance and reliability.

\section{ACKNOWLEDGMENT}

Thanks are due to the members of the magnet team at RAL (UK), CEA (F), KEK (J), INFN-LASA (I) and CERN.

\section{REFERENCES}

[1] ATLAS Magnet System Technical Design Report-4 Volumes, CERN document CERN/LHHC/97-18, 19, 20, 21, 1997.

[2] H. ten Kate et al., ATLAS Superconducting Magnet System: Survey of Construction, Test and Installation.

[3] P. Miele and $\mathrm{H}$. ten Kate, The superconducting magnet system for the ATLAS detector at CERN, in SOFT 21, Madrid, 2000.

[4] P. Vedrine et al., Manufacturing and Integration Progress of the ATLAS Barrel Toroid Air-Core Magnet at CERN.

[5] E. Baynham et al., ATLAS End Cap Toroid Magnets Cold Mass Design and Manufacturing Status.

[6] A. Yamamoto et al., "Progress in ATLAS central solenoid magnet," IEEE Trans. Appl. Supercond., vol. 10, no. 1, pp. 353-356, March 2000.

[7] N. Delruelle et al., "The Helium cryogenic system for the ATLAS experiment," IEEE Trans. Appl. Supercond., vol. 10, no. 1, pp. 1151-1153, March 2000.

[8] R. Ruber et al., On-Surface Integration and Test of the ATLAS Central Solenoid and Its Proximity Cryogenics.

[9] F. Haug et al., "The proximity cryogenic system for the ATLAS toroidal magnets," Adv. Cryo. Eng., vol. 47A, pp. 155-160, 2002.

[10] R. Pengo et al., "Test results of a $1.2 \mathrm{~kg} / \mathrm{s}$ centrifugal liquid Helium pump for the ATLAS superconducting toroid magnet system," in Proc. 19th Int. Cryo. Eng. Conf. (ICEC-19), G. Baguer et al., Ed., Narosa, 2003, pp. 71-74. 